\title{
Review Article \\ Simultaneous Minimally Invasive Treatment of Colorectal Neoplasm with Synchronous Liver Metastasis
}

\author{
Stefano Garritano, ${ }^{1}$ Federico Selvaggi, ${ }^{2}$ and Marcello Giuseppe Spampinato ${ }^{3}$ \\ ${ }^{1}$ General and Reconstructive Surgery, Department of Surgical Sciences, Policlinico Umberto I, "Sapienza” University of Rome, \\ 00161 Rome, Italy \\ ${ }^{2}$ Division of Surgery, Casa di Cura Villa Serena, Città Sant'Angelo, 65013 Pescara, Italy \\ ${ }^{3} \mathrm{HPB}$ and Advanced Minimally Invasive Liver Surgical Unit, Department of General and Minimally Invasive Surgery, \\ Policlinico Abano Terme, 1 Cristoforo Colombo Square, Abano Terme, 35031 Padua, Italy \\ Correspondence should be addressed to Marcello Giuseppe Spampinato; marcello.spampinato@gmail.com
}

Received 21 November 2015; Accepted 24 March 2016

Academic Editor: Dimitrios Dimitroulis

Copyright (C) 2016 Stefano Garritano et al. This is an open access article distributed under the Creative Commons Attribution License, which permits unrestricted use, distribution, and reproduction in any medium, provided the original work is properly cited.

\begin{abstract}
Purpose. To analyse perioperative and oncological outcomes of minimally invasive simultaneous resection of primary colorectal neoplasm with synchronous liver metastases. Methods. A Medline revision of the current published literature on laparoscopic and robotic-assisted combined colectomy with hepatectomy for synchronous liver metastatic colorectal neoplasm was performed until February 2015. The specific search terms were "liver metastases", "hepatic metastases", "colorectal”, "colon", "rectal", "minimally invasive", "laparoscopy", "robotic-assisted", "robotic colorectal and liver resection", "synchronous", and "simultaneous". Results. 20 clinical reports including 150 patients who underwent minimally invasive one-stage procedure were retrospectively analysed. No randomized trials were found. The approach was laparoscopic in 139 patients $(92.7 \%)$ and robotic in 11 cases $(7.3 \%)$. The rectum was the most resected site of primary neoplasm $(52.7 \%)$ and combined liver procedure was in $89 \%$ of cases a minor liver resection. One patient $(0.7 \%)$ required conversion to open surgery. The overall morbidity and mortality rate were $18 \%$ and $1.3 \%$, respectively. The most common complication was colorectal anastomotic leakage. Data concerning oncologic outcomes were too heterogeneous in order to gather definitive results. Conclusion. Although no prospective randomized trials are available, one-stage minimally invasive approach seems to show advantages over conventional surgery in terms of postoperative short-term course. On the contrary, more studies are required to define the oncologic values of the minimally invasive combined treatment.
\end{abstract}

\section{Introduction}

Colorectal cancer (CRC) is the third most commonly diagnosed cancer in males and the second in females, with an estimated 1.4 million cases and 693,900 deaths that occurred in 2012 in the world [1]. The highest incidence rates are reported in Western countries with an age-standardized rate of 36.3 per 100,000 for male and 23.6 per 100,000 for female in 2012 [1]. Survival is determined by tumor stage with 5year relative survival rates of $90.3 \%$ for Stage I and only $12.5 \%$ for stage IV [2]. The liver is the most common site of CRC metastasis, and synchronous liver metastases (SLMs) are found in up to $25 \%$ of CRC patients [3-7]. For these patients, a curative resection (R0) is the only therapeutic chance of long-term survival, although the problem of how to optimally schedule colorectal and liver operation plus neoadjuvant and/or adjuvant chemotherapy is still debated [8-12].

Different surgical strategies have been proposed to treat CRC with SLMs. Among these, the one-stage approach has been showed to be safe and effective as the classic colonfirst approach, even when major hepatectomies are required [13]. This was confirmed by a recent multicenter international study that compared simultaneous versus staged approaches showing no difference in morbidity and mortality rates as well as long-term outcomes [14]. Despite opponents, the laparoscopic approach to CRC and SLMs has been demonstrated to be effective by several studies [15-20]. Recently, the one-stage 
minimally invasive approach (MIA) has been showed to be as safe and effective as the open treatment even for simultaneous resection of CRC with SLMs [9, 21-24]. Besides, in the last decade the use of the robotic technology has been proposed by some authors in the field of colorectal and liver surgery as an alternative to laparoscopy, in order to overcome some technical limitations [25-27]. The aim of this review is to analyse current literature concerning one-stage MIA for CRC and SMLs patients, with particular emphasis on technical issues and perioperative and oncologic outcomes.

\section{Methods}

2.1. Inclusion and Exclusion Criteria. We analysed recent published studies that describe the clinical course of patients who underwent one-stage resection of CRC with SLMs totally by MIA, including laparoscopic or robotic-assisted procedures. Case reports and case series were retrospectively reviewed. An intention to treat analysis was applied and, therefore, cases converted to open procedures were included. On the contrary, cases treated with combined laparoscopy/laparotomy procedure (hybrid technique) or hand-assisted procedure were excluded. In addition, clinical conditions in which minimally invasive results are not clearly reported or not distinguished from conventional surgical outcomes were excluded. The Brisbane 2000 Terminology of Liver Anatomy and Resections was used to define minor or major liver resection [34]. According to this, anatomic and nonanatomic hepatic parenchymal resections, even in association with radiofrequency ablation (RFA), were included.

2.2. Medline Research Criteria. A systematic search was conducted using Medline (through PubMed) for all reports published until February 2015 (the last search was performed on February 28th). The search words for the literature review were scheduled in four groups:

(i) First group: "liver metastases" and "hepatic metastases".

(ii) Second group: "colorectal", "colon", and "rectal".

(iii) Third group: "minimally invasive", "laparoscopy", "robotic-assisted", and "robotic colorectal and liver resection".

(iv) Fourth group: "synchronous" and "simultaneous".

The search terms were designed by combining one word from each group, so that all possible combinations were employed. This process yielded 96 search terms, all of which were sought in titles and/or abstracts of English written papers. Results were enriched by 32 additional articles, manually searched or listed in the reference. Finally, two authors (Stefano Garritano and Federico Selvaggi) reviewed all abstracts independently and the full text of relevant studies was considered for inclusion.

\section{Results}

The research was brought to a total of 128 manuscripts of which only 49 were suitable for inclusion in the study. Of
TABLE 1: Demographic and clinical characteristics.

\begin{tabular}{lc}
\hline Variable & Patients n. 150 (\%) \\
\hline Age (years), range & $60(31-88)$ \\
ASA (n.) & $75(50 \%)$ \\
Unknown & $52(34.7 \%)$ \\
ASA 1-2 & $23(15.3 \%)$ \\
ASA 3-4 & \\
Location of primary tumor (n.) & $35(23.3 \%)$ \\
$\quad$ Right colon & $36(24 \%)$ \\
Left colon & $79(52.7 \%)$ \\
Rectum & \\
Number of liver metastases (n.) & $20(13.3 \%)$ \\
Unknown & $81(54 \%)$ \\
Single & $49(32.7 \%)$ \\
Multiple & \\
Location of SLMs (n.) & $131(87.3 \%)$ \\
Unilobar & $19(12.7 \%)$ \\
Bilobar & \\
Size of SLMs (n.) & $85(56.6 \%)$ \\
Unknown & $22(14.6 \%)$ \\
$\leq 2$ cm & $43(28.8 \%)$ \\
$>2$ cm &
\end{tabular}

ASA: American Society of Anesthesiologists classification and SLMs: synchronous liver metastases.

these, 29 papers were excluded according to the reported criteria, and finally a total of 20 papers were analysed (Figure 1). No randomized clinical trials were found. We assessed retrospective clinical data of 150 patients who underwent one-step MIA for primary CRC with SLMs.

3.1. Patients and Neoplasm Characteristics. Demographic and clinic-pathological data are reported in Table 1. Median age of patients was 60 years (range, 31-88). According to American Society of Anesthesiologists (ASA) grade, the patient health status was classified as ASA I-II in 52 (34.7\%) cases, ASA IIIIV in $23(15.3 \%)$ cases, and unknown in 75 (50\%) of cases. CRC was diagnosed in right colon in $35(23.3 \%)$ cases, left colon in $36(24 \%)$ cases, and rectum in 79 (52.7\%). SLMs were single in $81(54 \%)$ patients, multiple in $49(32.7 \%)$, and unknown in $20(13.3 \%)$ cases. Location of SLMs was unilobar in 131 (87.3\%) and bilobar in 19 (12.7\%), respectively. Eleven (7.3\%) patients underwent neoadjuvant chemotherapy (Table 2).

3.2. Operative Outcomes. Operative outcomes are shown in Tables 2 and 3. All procedures but one (0.7\%) were completed by MIA with no conversion to hybrid or open procedures. The approach was laparoscopic in 139 (92.7\%) cases and robotic-assisted in $11(7.3 \%)$ cases. According to CRC location, there were $35(23.3 \%)$ right colectomies, 36 (24\%) left colectomies/sigmoidectomies, 68 (45.3\%) anterior rectal resections (ARR), 10 (6.7\%) Miles procedures, and one $(0.7 \%)$ subtotal proctocolectomy. Temporary ileostomy was reported in $8(5.3 \%)$ patients who underwent ARR. Liver 


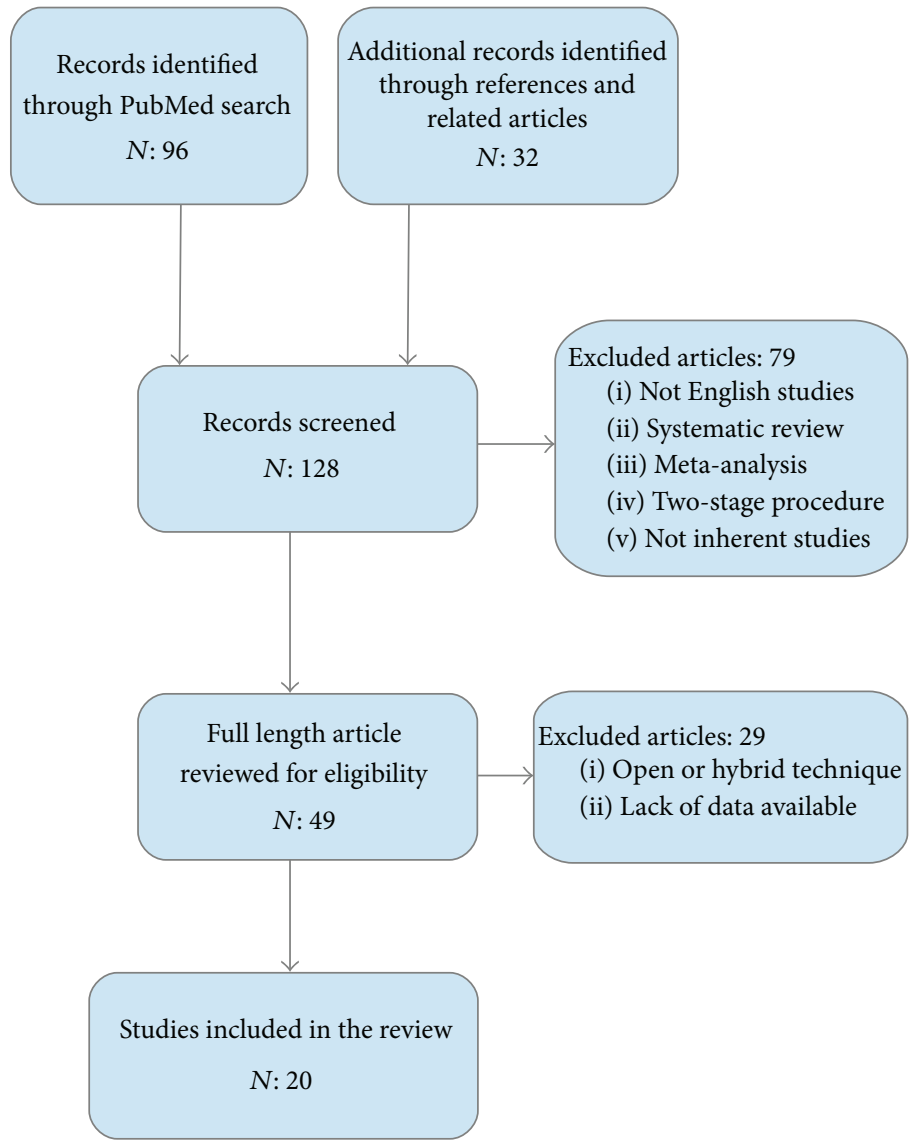

FIGURE 1: Flow chart showing selection of articles.

resection was minor in $134(89.3 \%)$ patients and major in 16 $(10.7 \%)$ cases, respectively. Liver resection was nonanatomical in $90(60 \%)$ cases and anatomical in $60(40 \%)$ cases. The first performed procedure was liver resection in $56(37 \%)$ cases and colorectal in $84(56 \%)$, while it was unknown in $10(7 \%)$ cases. Intermittent Pringle's Manoeuvre (IPM) was frequently prepared but finally used only in selected and limited conditions. Total median operative time was 320 minutes (range, 120-749 minutes). Median estimated blood loss was $259 \mathrm{~mL}$ (range, $10-1500 \mathrm{~mL}$ ) (Table 3).

3.3. Perioperative Outcomes. Overall morbidity and mortality rates were $18 \%$ and $1.3 \%$, respectively (Table 4 ). There was one patient who experienced uncontrolled bleeding that finally required conversion to open surgery. Postoperative complications are reported in Table 4 . The most common complication was colorectal anastomotic leakage (3.3\%). Median length of hospital stay was 8.5 days (range, 3-54 days). Perioperative mortality within 30 days was reported in 2 (1.3\%) cases (Table 4).

3.4. Oncologic Short-Term Outcomes. Only 14 authors have reported oncologic outcomes with recurrences in a cohort of 107 patients. Preoperative CRC diagnosis was confirmed by histology in 147 cases (data not included in the table). The SLMs diagnosis was not confirmed postoperatively in 5 cases as reported by two authors [10, 22]. There were $101(67.3 \%)$ cases of R0 status and 6 (4\%) cases of R1 and 43 (28.7\%) were the unknown status.

\section{Discussion}

Although the optimal strategy for resectable CRC with SLMs has not been established yet, the one-stage approach for simultaneous colectomy and hepatectomy gives the advantages to avoid two surgical procedures thus reducing risk for patient and costs for the community while keeping acceptable morbidity and good oncologic results $[13,35]$. Recently, MIA for simultaneous resection of CRC and SLMs has become popular [10, 29, 31, 33, 36]. Modern combined MIA, by laparoscopic and/or with robotic assistance, although being in its preliminary phase of experiences, has been showed to be feasible and safe even in cases requiring major liver resections $[21,25,30,36]$. In the present study perioperative outcomes of 150 patients, affected by stage IV CRC and SLMs treated by MIA up to February 2015, were retrospectively analysed. The reported data confirmed the feasibility and safety of simultaneous MIA with acceptable perioperative morbidity and mortality. Surprisingly, despite the high rate of ARR the number of temporary ileostomies was low $[6,10,22,28]$. This could be explained by the high rate of minor liver resections and the apparently infrequent use of IPM to perform it. In 


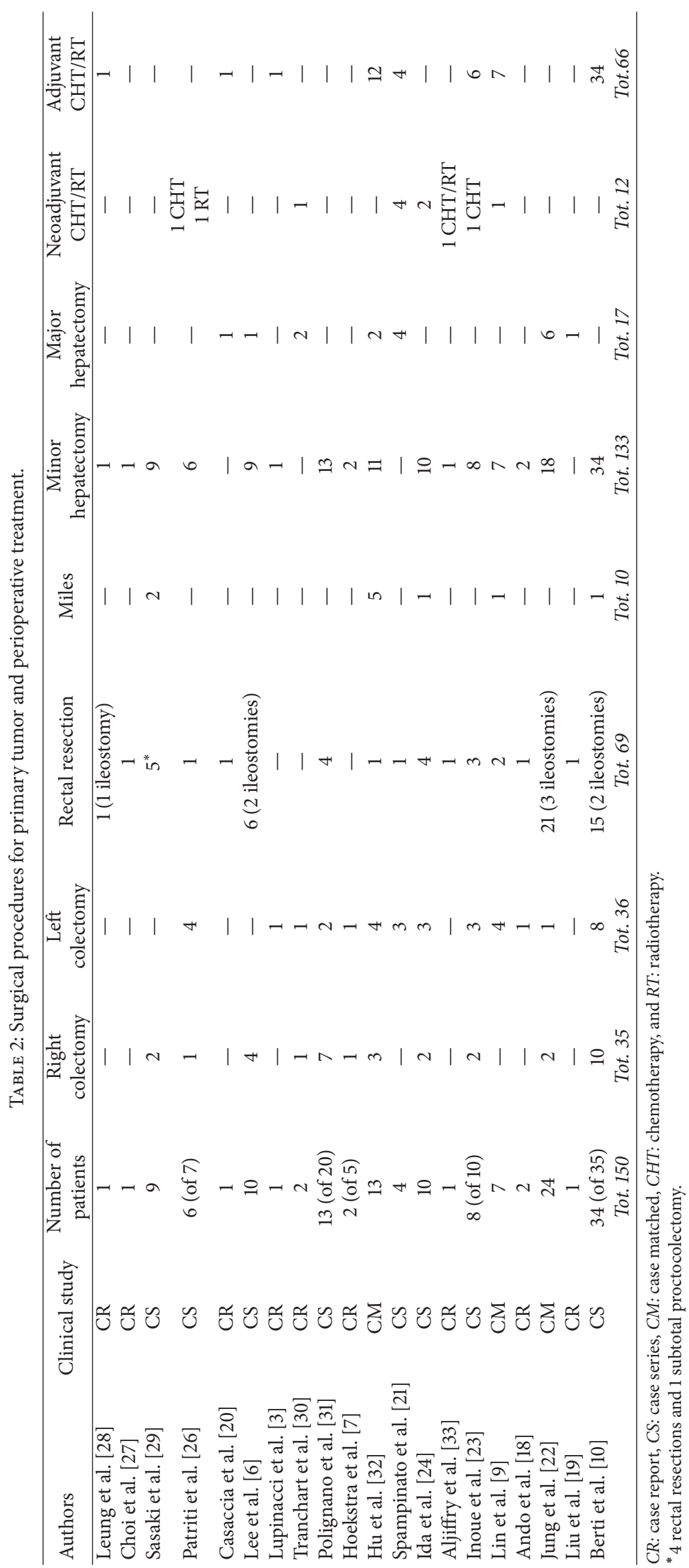


TABLE 3: Surgical outcomes.

\begin{tabular}{lc}
\hline Surgical procedure (n.) & $139(92.7 \%)$ \\
Totally laparoscopic & $11(7.3 \%)$ \\
Laparoscopic, robotic-assisted & \\
First step procedure (n.) & $10(7 \%)$ \\
Unknown & $56(37 \%)$ \\
Liver approach & $84(56 \%)$ \\
Colorectal approach & \\
Operation for primary tumor (n.) & $35(23.3 \%)$ \\
Right colectomy & $36(24 \%)$ \\
Left colectomy & $68(45.3 \%)$ \\
Anterior rectal resection & $10(6.7 \%)$ \\
Miles procedure & $1(0.7 \%)$ \\
Subtotal proctocolectomy & $8(5.3 \%)$ \\
Temporary ileostomy (n.) & \\
Hepatectomy (n.) & $134(89.3 \%)$ \\
Minor resection (<3 segments) & $16(10.7 \%)$ \\
Major resection ( $\geq 3$ segments) & $60(40 \%)$ \\
Anatomical resection & $90(60 \%)$ \\
Nonanatomical resection & $1(0.7 \%)$ \\
Conversion to laparotomy (n.) & $10(6.7 \%)$ \\
Intermittent Pringle’s Manoeuvre (n.) & $320($ range $120-749)$ \\
Operative time (min) & $259($ range $10-1500)$ \\
Estimated blood loss (mL) &
\end{tabular}

TABLE 4: Postoperative outcomes.

\begin{tabular}{lc}
\hline Overall morbidity (n.) & $27(18 \%)$ \\
Postoperative medical complications (n.) & $13(8.6 \%)$ \\
Thrombocytopenia & 1 \\
Postoperative ileus & 3 \\
Myocardial infarction & 2 \\
Pleural effusion & 2 \\
Pneumonia & 1 \\
MOF & 1 \\
CVC infection & 1 \\
Ictus cerebri & 1 \\
Deep vein thrombosis & 1 \\
Postoperative surgical complications (n.) & $14(9.3 \%)$ \\
Primary anastomotic leakage & 5 \\
Bile leakage & 3 \\
Liver abscess & 2 \\
Colovaginal fistula & 1 \\
Postoperative intestinal obstruction & 1 \\
Site infection & 1 \\
Unknown & 1 \\
Hospital stay (days), range & 8.5 (range 3-54) \\
30-day mortality (n.) & $2(1.3 \%)$ \\
\hline
\end{tabular}

fact, prolonged vascular clamping is responsible for transient portal hypertension with oedema of the intestinal mucosa that ultimately might be leading to colorectal anastomotic failure [21,37]. In this review, only 16 patients underwent minimally invasive colorectal resection associated with a major hepatectomy, confirming that this type of procedure is performed only by few specialized centers. In addition, most of the cases, including major and anatomical liver resection, were performed by two different specialized teams allowing good results in terms of conversion and perioperative outcomes. In fact, the morbidity and mortality rate were similar to the conventional open approach $[32,38]$. In more than $50 \%$ of cases, colorectal resection was the first procedure performed. Instead, as other authors, we believe that the choice of carrying out the liver resection as first step of treatment gives to the surgeon the opportunity to change surgical strategy from a combined procedure to a "liver first" resection which has been showed to be another effective treatment for stage IV CRC [39]. Indeed, this happened in one patient of our series, who showed intraoperatively chemotherapy related steatohepatitis, that finally developed transient lifethreading postoperative acute liver insufficiency [21]. In this review only five patients experienced colorectal anastomotic failure but we could not understand if this complication was associated with type of operation performed at first, or time of vascular clamping, prolonged operative time, and blood loss. Interestingly, only eleven patients underwent neoadjuvant chemotherapy prior to surgical therapy. The limited use of neoadjuvant chemotherapy along with a limited number of performed major hepatectomies could explain the overall low rate of complications found in this review. In fact, it is well known that chemotherapy associated steatohepatitis and volume of resected liver are associated with an increased number of post-liver-resection complications, including bleeding and liver failure [40]. Unfortunately, data regarding overall survival and recurrence were too heterogeneous to understand whether or not preoperative chemotherapy affected the oncologic results in terms of overall survival and recurrences. Other limits of this retrospective analysis were the difficulties in resuming data concerning the microscopic diffusion of the disease, such as distance of resection margins, number of harvested lymph nodes, and tumor stage classification. These criticisms reflect the nonhomogeneity of reported data, concerning R0 status or local and distal recurrence of the disease. However, two retrospectives studies have reported no differences in terms of overall survival, while a faster surgical recovery was observed for MIA patients $[9,32]$.

In order to overcome the limitations of laparoscopy, some authors have advocated the use of the da Vinci® ${ }^{\circledR}$ surgical system for liver resection [41, 42]. Wristed instruments offering seven degrees of freedom, tremor filtration with stereoscopic steady view, together with optimal working ergonomics, and avoidance of "fulcrum effect" are the main strengths of the robotic platform. No doubt exists about the fact that these technological improvements enhance surgical dexterity [43]. Recently, some authors have demonstrated that an increased number of patients can receive a laparoscopic major hepatectomy, especially when robotic assistance was used $[41,44]$. However, in our review the majority of cases was performed laparoscopically and the use of the robotic platform was limited. These data might reflect the infancy of the robotic technology and the related costs of the procedure [45]. 


\section{Conclusions}

One-stage MIA for CRC patients with SLMs can be performed safely with an acceptable morbidity. Reduced hospital stay and the faster enrolment of patients to adjuvant treatments represent the most relevant advantages of MIA compared with conventional surgery. Indeed, MIA offers all the benefits of laparoscopic minimally invasiveness, especially in terms of fewer postoperative adhesions. This represents a crucial aspect when redo liver surgery is indicated for recurrences. With all the criticisms of a retrospective and noncomparative analysis, this review showed that MIA for simultaneous colectomy and hepatectomy can be performed safely even in cases requiring major liver resections, when the combined procedures are performed by specialized and well trained teams. Prospective and randomized trials are needed to define the oncological benefits and validate the role of onestage MIA for CRC patients with SLMs.

\section{Abbreviations}

CRC: Colorectal cancer

SLMs: Synchronous liver metastases

MIA: Minimally invasive approach

IPM: Intermittent Pringle's Manoeuvre.

\section{Competing Interests}

Stefano Garritano and other coauthors have no competing interests.

\section{References}

[1] L. A. Torre, F. Bray, R. L. Siegel, J. Ferlay, J. Lortet-Tieulent, and A. Jemal, "Global cancer statistics, 2012," CA Cancer Journal for Clinicians, vol. 65, no. 2, pp. 87-108, 2015.

[2] R. Siegel, C. Desantis, and A. Jemal, "Colorectal cancer statistics, 2014," CA: A Cancer Journal for Clinicians, vol. 64, no. 2, pp. 104117, 2014.

[3] R. M. Lupinacci, M. A. Machado, R. A. Lupinacci, and P. Herman, "Simultaneous left colectomy and standard hepatectomy reformed by laparoscopy," Revista do Colégio Brasileiro de Cirurgiões, vol. 38, no. 2, pp. 139-141, 2011.

[4] R. Mantke, U. Schmidt, S. Wolff, R. Kube, and H. Lippert, "Incidence of synchronous liver metastases in patients with colorectal cancer in relationship to clinico-pathologic characteristics. Results of a German prospective multicentre observational study," European Journal of Surgical Oncology, vol. 38, no. 3, pp. 259-265, 2012.

[5] L. Wang, H. M. Tan, J. W. Tan, Y. Chu, X. M. Ding, and J. H. Dong, "Laparoscopic approach for simultaneous treatment of rectal cancer and synchronous liver metastases: indications and initial experience," Hepato-Gastroenterology, vol. 60, no. 121, pp. 217-221, 2013.

[6] J.-S. Lee, H.-T. Hong, J.-H. Kim et al., "Simultaneous laparoscopic resection of primary colorectal cancer and metastatic liver tumor: initial experience of single institute," Journal of Laparoendoscopic and Advanced Surgical Techniques, vol. 20, no. 8, pp. 683-687, 2010.
[7] L. T. Hoekstra, O. R. C. Busch, W. A. Bemelman, T. M. Van Gulik, and P. J. Tanis, "Initial experiences of simultaneous laparoscopic resection of colorectal cancer and liver metastases," HPB Surgery, vol. 2012, Article ID 893956, 6 pages, 2012.

[8] R. L. C. Araujo, M. Gönen, and P. Herman, "Chemotherapy for patients with colorectal liver metastases who underwent curative resection improves long-term outcomes: systematic review and meta-analysis," Annals of Surgical Oncology, vol. 22, no. 9, pp. 3070-3078, 2015.

[9] Q. Lin, Q. Ye, D. Zhu et al., "Comparison of minimally invasive and open colorectal resections for patients undergoing simultaneous R0 resection for liver metastases: a propensity score analysis," International Journal of Colorectal Disease, vol. 30, no. 3, pp. 385-395, 2015.

[10] S. Berti, E. Francone, M. Minuto et al., "Synchronous totally laparoscopic management of colorectal cancer and resectable liver metastases: a single center experience," Langenbeck's Archives of Surgery, vol. 400, no. 4, pp. 495-503, 2015.

[11] J. A. Castellanos and N. B. Merchant, "Strategies for management of synchronous colorectal metastases," Current Surgery Reports, vol. 2, article 62, 2014.

[12] G. Veereman, J. Robays, L. Verleye et al., "Pooled analysis of the surgical treatment for colorectal cancer liver metastases," Critical Reviews in Oncology/Hematology, vol. 94, no. 1, pp. 122135, 2015

[13] L. Capussotti, A. Ferrero, L. Viganò, D. Ribero, R. L. Tesoriere, and R. Polastri, "Major liver resections synchronous with colorectal surgery," Annals of Surgical Oncology, vol. 14, no. 1, pp. 195-201, 2007.

[14] S. C. Mayo, C. Pulitano, H. Marques et al., "Surgical management of patients with synchronous colorectal liver metastasis: a multicenter international analysis," Journal of the American College of Surgeons, vol. 216, no. 4, pp. 707-718, 2013.

[15] A. M. Lacy, S. Delgado, A. Castells et al., “The long-term results of a randomized clinical trial of laparoscopy-assisted versus open surgery for colon cancer," Annals of Surgery, vol. 248, no. 1, pp. 1-7, 2008.

[16] K. T. Nguyen, A. Laurent, I. Dagher et al., "Minimally invasive liver resection for metastatic colorectal cancer: a multiinstitutional, international report of safety, feasibility, and early outcomes," Annals of Surgery, vol. 250, no. 5, pp. 842-848, 2009.

[17] K. T. Nguyen, J. W. Marsh, A. Tsung, J. J. L. Steel, T. C. Gamblin, and D. A. Geller, "Comparative benefits of laparoscopic vs open hepatic resection: a critical appraisal," Archives of Surgery, vol. 146, no. 3, pp. 348-356, 2011.

[18] K. Ando, E. Oki, T. Ikeda et al., "Simultaneous resection of colorectal cancer and liver metastases in the right lobe using pure laparoscopic surgery," Surgery Today, vol. 44, no. 8, pp. 1588-1592, 2014.

[19] L. Liu, Y. Zeng, W. Lai et al., "Laparoscopic anterior approach of major hepatectomy combined with colorectal resection for synchronous colorectal liver metastases," Surgical Laparoscopy, Endoscopy and Percutaneous Techniques, vol. 24, no. 6, pp. e237e240, 2014.

[20] M. Casaccia, F. Famiglietti, E. Andorno, S. Di Domenico, C. Ferrari, and U. Valente, "Simultaneous laparoscopic anterior resection and left hepatic lobectomy for stage IV rectal cancer," Journal of the Society of Laparoendoscopic Surgeons, vol. 14, no. 3, pp. 414-417, 2010.

[21] M. G. Spampinato, L. Mandalá, G. Quarta, P. Del Medico, and G. Baldazzi, "One-stage, totally laparoscopic major hepatectomy 
and colectomy for colorectal neoplasm with synchronous liver metastasis: safety, feasibility and short-term outcome," Surgery, vol. 153, no. 6, pp. 861-865, 2013.

[22] K. U. Jung, H. C. Kim, Y. B. Cho et al., "Outcomes of simultaneous laparoscopic colorectal and hepatic resection for patients with colorectal cancers: a comparative study," Journal of Laparoendoscopic and Advanced Surgical Techniques, vol. 24, no. 4, pp. 229-235, 2014.

[23] A. Inoue, M. Uemura, H. Yamamoto et al., "Short-term outcomes of simultaneous laparoscopic colectomy and hepatectomy for primary colorectal cancer with synchronous liver metastases," International Surgery, vol. 99, no. 4, pp. 338-343, 2014.

[24] S. Ida, E. Oki, K. Ando et al., "Pure laparoscopic right-sided hepatectomy in the semi-prone position for synchronous colorectal cancer with liver metastases," Asian Journal of Endoscopic Surgery, vol. 7, no. 2, pp. 133-137, 2014.

[25] G. H. Choi, S. H. Choi, S. H. Kim et al., "Robotic liver resection: technique and results of 30 consecutive procedures," Surgical Endoscopy and Other Interventional Techniques, vol. 26, no. 8, pp. 2247-2258, 2012.

[26] A. Patriti, G. Ceccarelli, A. Bartoli, A. Spaziani, L. M. Lapalorcia, and L. Casciola, "Laparoscopic and robot-assisted onestage resection of colorectal cancer with synchronous liver metastases: a pilot study," Journal of Hepato-Biliary-Pancreatic Surgery, vol. 16, no. 4, pp. 450-457, 2009.

[27] S. B. Choi, J. S. Park, J. K. Kim et al., "Early experiences of robotic-assisted laparoscopic liver resection," Yonsei Medical Journal, vol. 49, no. 4, pp. 632-638, 2008.

[28] K. L. Leung, J. F. Lee, R. Y. Yiu, S. S. Ng, and J. C. Li, "Simultaneous laparoscopic resection of rectal cancer and liver metastasis," Journal of Laparoendoscopic and Advanced Surgical Techniques A, vol. 16, no. 5, pp. 486-488, 2006.

[29] A. Sasaki, H. Nitta, K. Otsuka, T. Takahara, S. Nishizuka, and G. Wakabayashi, "Ten-year experience of totally laparoscopic liwer resection in a single institution," British Journal of Surgery, vol. 96, no. 3, pp. 274-279, 2009.

[30] H. Tranchart, P. S. Diop, P. Lainas et al., "Laparoscopic major hepatectomy can be safely performed with colorectal surgery for synchronous colorectal liver metastasis," $H P B$, vol. 13, no. 1, pp. 46-50, 2011.

[31] F. M. Polignano, A. J. Quyn, P. Sanjay, N. A. Henderson, and I. S. Tait, "Totally laparoscopic strategies for the management of colorectal cancer with synchronous liver metastasis," Surgical Endoscopy and Other Interventional Techniques, vol. 26, no. 9, pp. 2571-2578, 2012.

[32] M.-G. Hu, C.-G. Ou-Yang, G.-D. Zhao, D.-B. Xu, and R. Liu, "Outcomes of open versus laparoscopic procedure for synchronous radical resection of liver metastatic colorectal cancer: a comparative study," Surgical Laparoscopy, Endoscopy and Percutaneous Techniques, vol. 22, no. 4, pp. 364-369, 2012.

[33] M. Aljiffry, M. Alrajraji, S. Al-Sabah, and M. Hassanain, "Laparoscopic-assisted one-stage resection of rectal cancer with synchronous liver metastasis utilizing a pfannenstiel incision," Saudi Journal of Gastroenterology, vol. 20, no. 5, pp. 315-318, 2014.

[34] J. Belghiti, P.-A. Clavien, E. Gadzijev et al., “The Brisbane 2000 terminology of liver anatomy and resections," $H P B$, vol. 2, no. 3 , pp. 333-339, 2000.

[35] D. E. Abbott, S. B. Cantor, C.-Y. Hu et al., "Optimizing clinical and economic outcomes of surgical therapy for patients with colorectal cancer and synchronous liver metastases," Journal of the American College of Surgeons, vol. 215, no. 2, pp. 262-270, 2012.

[36] R. M. Lupinacci, W. Andraus, L. B. De Paiva Haddad, L. A. Carneiro D'Albuquerque, and P. Herman, "Simultaneous laparoscopic resection of primary colorectal cancer and associated liver metastases: a systematic review," Techniques in Coloproctology, vol. 18, no. 2, pp. 129-135, 2014.

[37] D. Jaeck, P. Bachellier, J. C. Weber et al., "Surgical strategy in the treatment of synchronous hepatic metastases of colorectal cancers. Analysis of a series of 59 operated on patients," Chirurgie, vol. 124, no. 3, pp. 258-263, 1999.

[38] E. De Santibañes, D. Fernandez, C. Vaccaro et al., "Shortterm and long-term outcomes after simultaneous resection of colorectal malignancies and synchronous liver metastases," World Journal of Surgery, vol. 34, no. 9, pp. 2133-2140, 2010.

[39] G. Mentha, A. D. Roth, S. Terraz et al., "'Liver first' approach in the treatment of colorectal cancer with synchronous liver metastases," Digestive Surgery, vol. 25, no. 6, pp. 430-435, 2009.

[40] A. Z. Khan, G. Morris-Stiff, and M. Makuuchi, "Patterns of chemotherapy-induced hepatic injury and their implications for patients undergoing liver resection for colorectal liver metastases," Journal of Hepato-Biliary-Pancreatic Surgery, vol. 16, no. 2, pp. 137-144, 2009.

[41] A. Tsung, D. A. Geller, D. C. Sukato et al., "Robotic versus laparoscopic hepatectomy: a matched comparison," Annals of Surgery, vol. 259, no. 3, pp. 549-555, 2014.

[42] P. C. Giulianotti, F. Sbrana, A. Coratti et al., "Totally robotic right hepatectomy: surgical technique and outcomes," Archives of Surgery, vol. 146, no. 7, pp. 844-850, 2011.

[43] K. Moorthy, Y. Munz, A. Dosis et al., "Dexterity enhancement with robotic surgery," Surgical Endoscopy and Other Interventional Techniques, vol. 18, no. 5, pp. 790-795, 2004.

[44] U. Boggi, S. Signori, N. De Lio et al., "Feasibility of robotic pancreaticoduodenectomy," The British Journal of Surgery, vol. 100, no. 7, pp. 917-925, 2013.

[45] M. G. Spampinato, A. Coratti, L. Bianco et al., "Perioperative outcomes of laparoscopic and robot-assisted major hepatectomies: an Italian multi-institutional comparative study," Surgical Endoscopy, vol. 28, no. 10, pp. 2973-2979, 2014. 


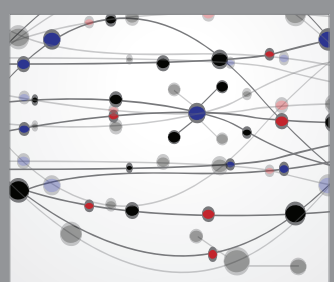

The Scientific World Journal
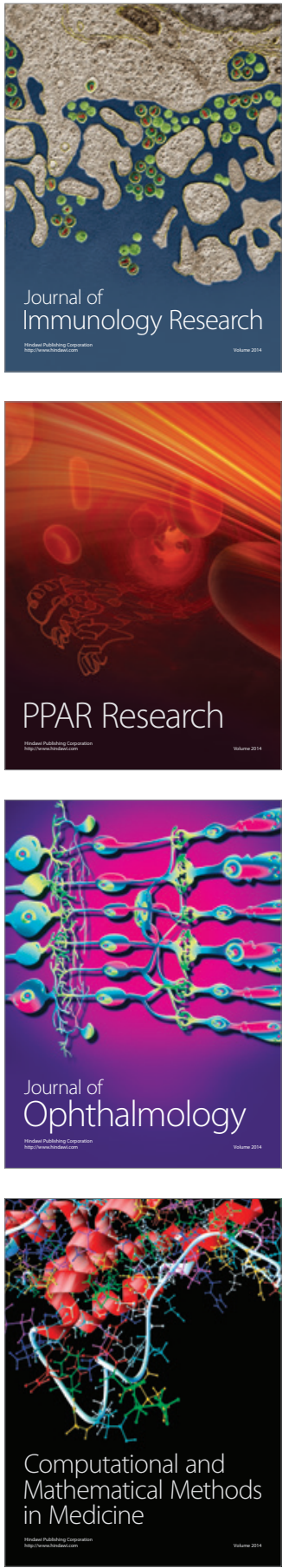

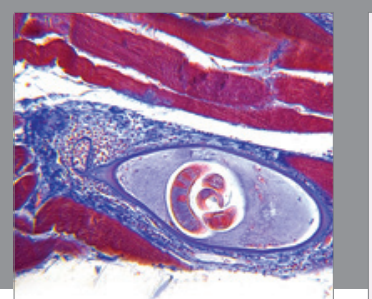

Gastroenterology Research and Practice

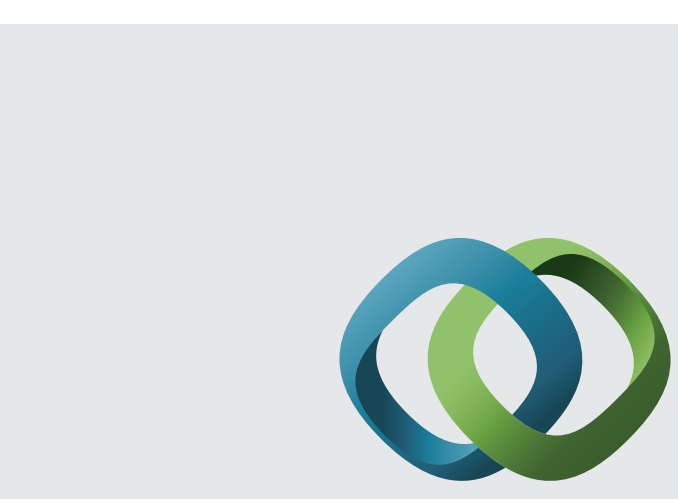

\section{Hindawi}

Submit your manuscripts at

http://www.hindawi.com
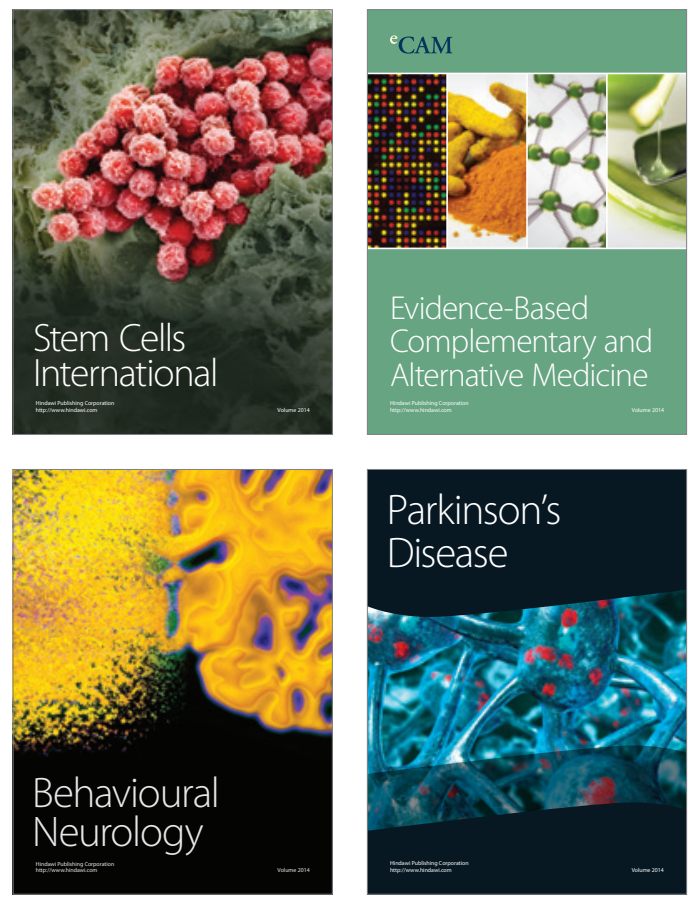
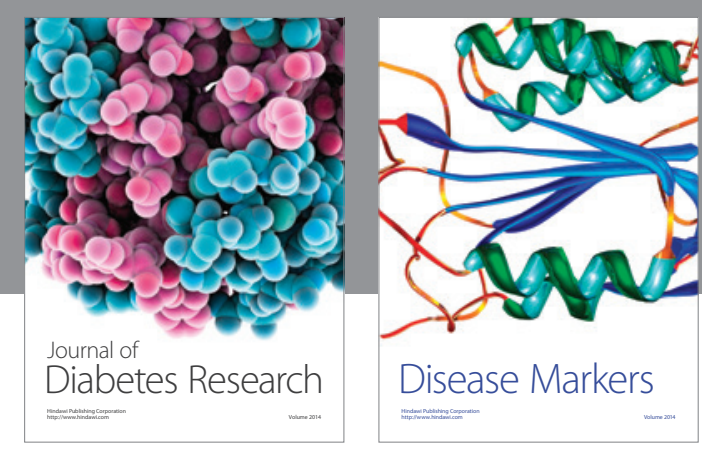

Disease Markers
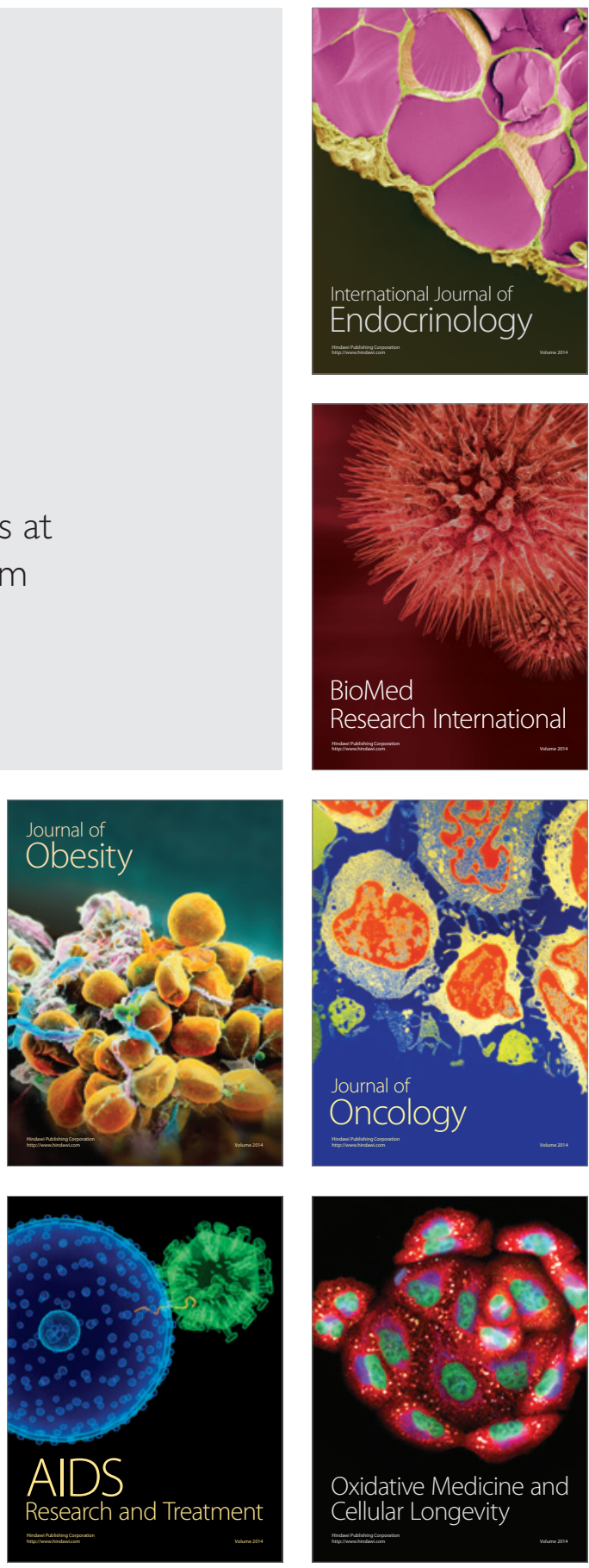